\title{
Horizonless Rotating Solutions in $(n+1)$-dimensional Einstein-Maxwell Gravity
}

\author{
M. H. Dehghant* \\ Physics Department and Biruni Observatory, \\ Shiraz University, Shiraz 71454, Iran \\ and \\ Institute for Studies in Theoretical Physics and Mathematics (IPM) \\ P.O. Box 19395-5531, Tehran, Iran
}

\begin{abstract}
We introduce two classes of rotating solutions of Einstein-Maxwell gravity in $n+1$ dimensions which are asymptotically anti-de Sitter type. They have no curvature singularity and no horizons. The first class of solutions, which has a conic singularity yields a spacetime with a longitudinal magnetic field and $k$ rotation parameters. We show that when one or more of the rotation parameters are non zero, the spinning brane has a net electric charge that is proportional to the magnitude of the rotation parameters. The second class of solutions yields a spacetime with an angular magnetic field and $\kappa$ boost parameters. We find that the net electric charge of these traveling branes with one or more nonzero boost parameters is proportional to the magnitude of the velocity of the brane. We also use the counterterm method inspired by AdS/CFT correspondence and calculate the conserved quantities of the solutions. We show that the logarithmic divergencies associated to the Weyl anomalies and matter field are zero, and the $r$ divergence of the action can be removed by the counterterm method.
\end{abstract}

\section{INTRODUCTION}

The anti-de Sitter conformal field theory (AdS/CFT) correspondence [1], which relates the low energy limit of string theory in asymptotically anti de-Sitter (AAdS) spacetime and

*Electronic address: dehghani@physics.susc.ac.ir 
the quantum field theory living on the boundary of it, have attracted a great deal of attention in recent years. This equivalence between the two formulations means that, at least in principle, one can obtain complete information on one side of the duality by performing computation on the other side. A dictionary translating between different quantities in the bulk gravity theory and its counterparts on the boundary has emerged, including the partition functions of both theories. An interesting application of the AdS/CFT correspondence is the interpretation of the Hawking-Page phase transition between a thermal AdS and AAdS black hole as the confinement-deconfinement phases of the Yang-Mills (dual gauge) theory defined on the AdS boundary [2]. This conjecture is now a fundamental concept that furnishes a means for calculating the action and conserved quantities intrinsically without reliance on any reference spacetime $[3,4,5]$. It has also been applied to the case of black holes with constant negative or zero curvature horizons [6] and rotating higher genus black branes [7]. Although the AdS/CFT correspondence applies for the case of a spatially infinite boundary, it was also employed for the computation of the conserved and thermodynamic quantities in the case of a finite boundary [8]. The counterterm method also has been extended to the case of asymptotically de Sitter spacetimes [9].

Thus, it has become important to obtain new solutions of the Einstein gravity with a negative cosmological constant and apply the AdS/CFT correspondence to them. For AAdS spacetimes, the presence of a negative cosmological constant makes it possible to have a large variety of static and stationary solutions with planar, spherical, or cylindrical symmetries. Asymptotically AdS black holes whose event horizons are hypersurfaces with a positive, negative, or zero scalar curvature were considered in [10]. Asymptotically AdS rotating topological black brane solutions have also been obtained [11]. The AAdS rotating solution of Einstein's equation with cylindrical and toroidal horizons and its extension to include the electromagnetic field were considered in Ref. [12]. The generalization of this AAdS charged rotating solution of the Einstein-Maxwell equation to higher dimensions and higher derivative gravity and investigation of its thermodynamics were done in [13, 14]. In the context of gauged supergravity models, the rotating brane solution has been considered by many authors (see, for example, [15]).

In this paper we are dealing with the issue of the spacetimes generated by spinning string/brane sources in $(n+1)$-dimensional Einstein-Maxwell theory that are horizonless and have nontrivial external solutions. These kinds of solutions have been investigated by many 
authors in four dimensions. Static uncharged cylindrically symmetric solutions of Einstein gravity in four dimensions were considered in [16]. Similar static solutions in the context of cosmic string theory were found in [17]. All of these solutions [16, 17] are horizonless and have a conical geometry; they are everywhere flat except at the location of the line source. The extension to include the electromagnetic field has also been done [18, 19]. Some solutions of type IIB supergravity compactified on a four dimensional torus were considered in [20], which have no curvature singularity and no conic singularity. Here we will generalize the four-dimensional solution found in [19] to the case of the $(n+1)$-dimensional solution with all rotation and boost parameters, and use the AdS/CFT correspondence to compute the conserved quantities of the system.

The outline of our paper is as follows. In Sec. III we first review the field equations of Einstein-Maxwell gravity and then introduce two classes of $(n+1)$-dimensional AAdS horizonless charged rotating solutions with more rotation and boost parameters. In Sec. III. we give a brief review of AdS/CFT correspondence, and obtain the logarithmic divergences associated with the Weyl anomalies and matter fields. The electric charge and conserved quantities of the system will also be computed. We finish our paper with some concluding remarks.

\section{II. (N+1)-DIMENSIONAL ROTATING SOLUTIONS OF EINSTEIN-MAXWELL GRAVITY}

\section{A. Field equations}

The gravitational action for Einstein-Maxwell theory in $n+1$ dimensions for AAdS spacetimes is

$$
I_{G}=-\frac{1}{16 \pi} \int_{\mathcal{M}} d^{n+1} x \sqrt{-g}\left(R-2 \Lambda-F^{\mu \nu} F_{\mu \nu}\right)+\frac{1}{8 \pi} \int_{\partial \mathcal{M}} d^{n} x \sqrt{-\gamma} K(\gamma),
$$

where $F_{\mu \nu}=\partial_{\mu} A_{\nu}-\partial_{\nu} A_{\mu}$ is the electromagnetic tensor field and $A_{\mu}$ is the vector potential. The first term is the Einstein-Hilbert volume term with negative cosmological constant $\Lambda=-n(n-1) / 2 l^{2}$ and the second term is the Gibbons-Hawking boundary term which is chosen such that the variational principle is well defined. The manifold $\mathcal{M}$ has metric $g_{\mu \nu}$ and covariant derivative $\nabla_{\mu}$. K is the trace of the extrinsic curvature $K^{\mu \nu}$ of any boundary(ies) $\partial \mathcal{M}$ of the manifold $\mathcal{M}$, with induced metric(s) $\gamma_{i, j}$. 
Varying the action over the metric tensor $g_{\mu \nu}$ and electromagnetic field $F_{\mu \nu}$, the equations of gravitational and electromagnetic fields are obtained as

$$
\begin{gathered}
R_{\mu \nu}-\frac{1}{2} g_{\mu \nu} R-\frac{n(n-1)}{2 l^{2}} g_{\mu \nu}=T_{\mu \nu} \\
\nabla_{\mu} F_{\mu \nu}=0
\end{gathered}
$$

where $T_{\mu \nu}^{e m}$ is the electromagnetic stress tensor

$$
T_{\mu \nu}^{e m}=2 F_{\mu}^{\lambda} F_{\lambda \nu}-\frac{1}{2} F_{\lambda \sigma} F^{\lambda \sigma} g_{\mu \nu}
$$

Now we want to obtain spacetimes generated by brane sources in $(n+1)$ dimensions which satisfy the above field equations. Some solutions were obtained in [21]. We will introduce two new classes of horizonless solutions to Eqs. (2)-(4), which are a generalization of those given by Dias and Lemos [19].

\section{B. Longitudinal magnetic field solutions with one rotation parameter}

It is a matter of calculation to show that the generalization of the four-dimensional solution given by Dias and Lemos [19] in $n+1$ dimensions with one rotation parameter can be written as

$$
d s^{2}=-\frac{\rho^{2}}{l^{2}}(\Xi d t-a d \phi)^{2}+f(\rho)\left(\frac{a}{l} d t-\Xi l d \phi\right)^{2}+\frac{d \rho^{2}}{f(\rho)}+\frac{\rho^{2}}{l^{2}} d X^{2},
$$

where $\Xi=\sqrt{1+a^{2} / l^{2}}, d X^{2}=\sum_{i=1}^{n-2}\left(d x^{i}\right)^{2}$ is the Euclidean metric on the $(n-2)$ dimensional submanifold, and $f(\rho)$ is

$$
f(\rho)=\frac{\rho^{2}}{l^{2}}+\frac{8 m l^{n-2}}{\rho^{n-2}}-\frac{8}{(n-1)(n-2)} \frac{q^{2} l^{2 n-4}}{\rho^{2 n-4}} .
$$

The gauge potential is given by

$$
A_{\mu}=\frac{2}{(n-2)} \frac{q l^{(n-3)}}{\rho^{n-2}}\left(a \delta_{\mu}^{0}-\Xi l^{2} \delta_{\mu}^{\phi}\right) .
$$

As we will see in Sec. III $m$ and $q$ in Eqs. (15)-(17) are the mass and charge parameters of the metric which are related to the mass and charge densities of the brane.

In order to study the general structure of this solution, we first look for curvature singularities. It is easy to show that the Kretschmann scalar $R_{\mu \nu \lambda \kappa} R^{\mu \nu \lambda \kappa}$ diverges at $\rho=0$ and 
therefore one might think that there is a curvature singularity located at $\rho=0$. However, as will be seen below, the spacetime will never achieve $\rho=0$. Now we look for the existence of horizons, and therefore we look for possible black brane solutions. One should conclude that there are no horizons and therefore no black brane solutions. The horizons, if any exist, are given by the zeros of the function $f(\rho)=g_{\rho \rho}^{-1}$. Let us denote the zeros of $f(\rho)$ by $r_{+}$. The function $f(\rho)$ is negative for $\rho<r_{+}$and positive for $\rho>r_{+}$, and therefore one may think that the hypersurface of constant time and $\rho=r_{+}$is the horizon. However, this analysis is not correct. Indeed, one may note that $g_{\rho \rho}$ and $g_{\phi \phi}$ are related by $f(\rho)=g_{\rho \rho}^{-1}=l^{-2} g_{\phi \phi}$, and therefore when $g_{\rho \rho}$ becomes negative (which occurs for $\rho<r_{+}$) so does $g_{\phi \phi}$. This leads to an apparent change of signature of the metric from $(n-1)+$ to $(n-2)+$, and therefore indicates that we are using an incorrect extension. To get rid of this incorrect extension, we introduce the new radial coordinate $r$ as

$$
r^{2}=\rho^{2}-r_{+}^{2} \Rightarrow d \rho^{2}=\frac{r^{2}}{r^{2}+r_{+}^{2}} d r^{2} .
$$

With this new coordinate, the metric (5) is

$$
\begin{aligned}
d s^{2}= & -\frac{r^{2}+r_{+}^{2}}{l^{2}}(\Xi d t-a d \phi)^{2}+f(r)\left(\frac{a}{l} d t-\Xi l d \phi\right)^{2} \\
& +\frac{r^{2}}{\left(r^{2}+r_{+}^{2}\right) f(r)} d r^{2}+\frac{r^{2}+r_{+}^{2}}{l^{2}} d X^{2},
\end{aligned}
$$

where the coordinates $r$ and $\phi$ assume the values $0 \leq r<\infty$ and $0 \leq \phi<2 \pi$, and $f(r)$ is now given as

$$
f(r)=\frac{r^{2}+r_{+}^{2}}{l^{2}}+\frac{8 m l^{n-2}}{\left(r^{2}+r_{+}^{2}\right)^{(n-2) / 2}}-\frac{8}{(n-1)(n-2)} \frac{q^{2} l^{2 n-4}}{\left(r^{2}+r_{+}^{2}\right)^{n-2}} .
$$

The gauge potential in the new coordinate is

$$
A_{\mu}=\frac{2}{(n-2)} \frac{q l^{(n-3)}}{\left(r^{2}+r_{+}^{2}\right)^{(n-2) / 2}}\left(a \delta_{\mu}^{0}-\Xi l^{2} \delta_{\mu}^{\phi}\right) .
$$

The function $f(r)$ given in Eq. (92) is positive in the whole spacetime and is zero at $r=0$. Also note that the Kretschmann scalar does not diverge in the range $0 \leq r<\infty$. Therefore this spacetime has no curvature singularities and no horizons. However, it has a conic geometry and has a conical singularity at $r=0$, since:

$$
\lim _{r \rightarrow 0} \frac{1}{r} \sqrt{\frac{g_{\phi \phi}}{g_{r r}}}=\frac{n h}{2 l}+\frac{4 q^{2}}{n-1}\left(\frac{l}{h}\right)^{2 n-3} \neq 1 .
$$


That is, as the radius $r$ tends to zero, the limit of the ratio "circumference/radius" is not $2 \pi$ and therefore the spacetime has a conical singularity at $r=0$. Of course, one may ask for the completeness of the spacetime with $r \geq 0$ (or $\rho \geq r_{+}$). It is easy to see that the spacetime described by Eq. (8) is both null and timelike geodesically complete (see the Appendix for more detail).

\section{Longitudinal magnetic field solutions with more rotation parameters}

The rotation group in $n+1$ dimensions is $S O(n)$ and therefore the number of independent rotation parameters is $[(n+1) / 2]$, where $[x]$ is the integer part of $x$. We now generalize the above solution given in Eq. (51) with $k \leq[(n+1) / 2]$ rotation parameters. This generalized solution can be written as

$$
\begin{aligned}
d s^{2}= & -\frac{r^{2}+r_{+}^{2}}{l^{2}}\left(\Xi d t-\sum_{i=1}^{k} a_{i} d \phi^{i}\right)^{2}+f(r)\left(\sqrt{\Xi^{2}-1} d t-\frac{\Xi}{\sqrt{\Xi^{2}-1}} \sum_{i=1}^{k} a_{i} d \phi^{i}\right)^{2} \\
& +\frac{r^{2} d r^{2}}{\left(r^{2}+r_{+}^{2}\right) f(r)}+\frac{r^{2}+r_{+}^{2}}{l^{2}\left(\Xi^{2}-1\right)} \sum_{i<j}^{k}\left(a_{i} d \phi_{j}-a_{j} d \phi_{i}\right)^{2}+\frac{r^{2}+r_{+}^{2}}{l^{2}} d X^{2},
\end{aligned}
$$

where $\Xi=\sqrt{1+\sum_{i}^{k} a_{i}^{2} / l^{2}}, d X^{2}$ is the Euclidean metric on the $(n-k-1)$-dimensional submanifold and $f(r)$ is the same as $f(r)$ given in Eq. (91). The gauge potential is

$$
A_{\mu}=\frac{2}{(n-2)} \frac{q l^{(n-2)}}{\left(r^{2}+r_{+}^{2}\right)^{(n-2) / 2}}\left(\sqrt{\Xi^{2}-1} \delta_{\mu}^{0}-\frac{\Xi}{\sqrt{\Xi^{2}-1}} a_{i} \delta_{\mu}^{i}\right) ; \quad \text { (no sum on i). }
$$

Again this spacetime has no horizon and curvature singularity. However, it has a conical singularity at $r=0$. One should note that these solutions are different from those discussed in [13], which were electrically charged rotating black brane solutions.

\section{Angular magnetic field solutions with one boost parameter}

The generalization of four-dimensional spacetime with angular magnetic field to the case of an $n+1$-dimensional AAdS charged rotating brane with one boost parameter is

$$
\begin{aligned}
d s^{2}= & -\frac{r^{2}+r_{+}^{2}}{l^{2}}\left(\Xi d t-\frac{v}{l} d z\right)^{2}+f(r)\left(\frac{v}{l} d t-\Xi d z\right)^{2} \\
& +\frac{r^{2} d r^{2}}{\left(r^{2}+r_{+}^{2}\right) f(r)}+\left(r^{2}+r_{+}^{2}\right) d \Omega^{2}
\end{aligned}
$$


where $\Xi=\sqrt{1+v^{2} / l^{2}}, d \Omega^{2}=\sum_{i=1}^{n-2}\left(d \phi^{i}\right)^{2}$, and $f(r)$ is the same as before given in Eq. (99). The coordinates $r$ and $\phi^{i}$ 's assume the values $0 \leq r<\infty$ and $0 \leq \phi^{i} \leq 2 \pi$, and the gauge potential is given by

$$
A_{\mu}=\frac{2}{(n-2)} \frac{q l^{n-3}}{\left(r^{2}+r_{+}^{2}\right)^{(n-2) / 2}}\left(a \delta_{\mu}^{0}-\Xi l \delta_{\mu}^{z}\right) .
$$

Using the same arguments given for the case of longitudinal magnetic field solutions discussed in Sec. II B, one can show that this spacetime has no curvature singularity, no horizons and no conical singularity.

\section{E. Angular magnetic field solutions with more boost parameters}

In this section we introduce the solutions of the Einstein-Maxwell equation with no rotation parameter and $\kappa$ boost parameters. The maximum number of boost parameters can be $n-2$. In this case the solution can be written as

$$
\begin{aligned}
d s^{2}= & -\frac{r^{2}+r_{+}^{2}}{l^{2}}\left(\Xi d t-l^{-1} \sum_{i=1}^{\kappa} v_{i} d x^{i}\right)^{2}+f(r)\left(\sqrt{\Xi^{2}-1} d t-\frac{\Xi}{l \sqrt{\Xi^{2}-1}} \sum_{i=1}^{\kappa} v_{i} d x^{i}\right)^{2} \\
& +\frac{r^{2}+r_{+}^{2}}{l^{4}\left(\Xi^{2}-1\right)} \sum_{i<j}^{\kappa}\left(v_{i} d x_{j}-v_{j} d x_{i}\right)^{2}+\frac{r^{2} d r^{2}}{\left(r^{2}+r_{+}^{2}\right) f(r)}+\left(r^{2}+r_{+}^{2}\right) d \Omega^{2}
\end{aligned}
$$

where $\Xi=\sqrt{1+\sum_{i}^{\kappa} v_{i}^{2} / l^{2}}, d \Omega^{2}=\sum_{i=1}^{n-\kappa-1}\left(d \phi^{i}\right)^{2}$, and $f(r)$ is given in Eq. (91). The gauge potential is given by

$$
A_{\mu}=\frac{2}{(n-2)} \frac{\lambda l^{(n-2)}}{\left(r^{2}+r_{+}^{2}\right)^{(n-2) / 2}}\left(\sqrt{\Xi^{2}-1} \delta_{\mu}^{0}-\frac{\Xi}{l \sqrt{\Xi^{2}-1}} v_{i} \delta_{\mu}^{i}\right) ; \quad \text { (no sum on i). }
$$

Again this spacetime has no curvature singularity, no horizons, and no conical singularity.

\section{THE CONSERVED QUANTITIES OF MAGNETIC ROTATING BRANE}

It is well known that the gravitational action given in Eq. (II) diverges. A systematic method of dealing with this divergence is through the use of the counterterm method inspired by AdS/CFT correspondence. The AdS/CFT correspondence states that, if the metric near the conformal boundary $(x \rightarrow 0)$ can be expanded in the AAdS form,

$$
d s^{2}=\frac{d x^{2}}{l^{2} x^{2}}+\frac{1}{x^{2}} \gamma_{i j} d x^{i} d x^{j},
$$


with nondegenerate metric $\gamma$, then one may remove the divergent terms in the action by adding a counterterm action, $I_{\mathrm{ct}}$, which is a functional of the boundary curvature invariants. The counterterm for asymptotically AdS spacetimes up to seven dimensions is

$$
\begin{aligned}
I_{\mathrm{ct}}= & \frac{1}{8 \pi} \int_{\partial \mathcal{M}_{\infty}} d^{n} x \sqrt{-\gamma}\left\{\frac{n-1}{l}-\frac{l \Theta(n-3)}{2(n-2)} R\right. \\
& \left.-\frac{l^{3} \Theta(n-5)}{2(n-4)(n-2)^{2}}\left(R_{a b} R^{a b}-\frac{n}{4(n-1)} R^{2}\right)+\ldots\right\},
\end{aligned}
$$

where $R, R_{a b c d}$, and $R_{a b}$ are the Ricci scalar, Riemann, and Ricci tensors of the boundary metric $\gamma_{a b}$, and $\Theta(x)$ is the step function, which is equal to 1 for $x \geq 0$ and zero otherwise. Although other counterterms (of higher mass dimension) may be added to $I_{c t}$, they will make no contribution to the evaluation of the action or Hamiltonian due to the rate at which they decrease toward infinity, and we shall not consider them in our analysis here. These counterterms have been used by many authors for a wide variety of spacetimes, including Schwarzschild-AdS, topological Schwarzschild-AdS, Kerr-AdS, Taub-NUT-AdS, Taub-bolt-AdS, and Taub-bolt-Kerr-AdS [22].

Of course, for even $n$ one has logarithmic divergences in the partition function which can be related to the Weyl anomalies in dual conformal field theory [3]. These logarithmic divergences associated with the Weyl anomalies of the dual field theory for $n=4$ and $n=6$ are $[23]$

$$
\begin{aligned}
I_{\log }=-\frac{\ln \epsilon}{64 \pi l^{3}} \int d^{4} x \sqrt{-\gamma^{0}}\left[\left(R_{i j}^{0}\right) R^{(0) i j}-\frac{1}{3}\left(R^{0}\right)^{2}\right], \\
I_{\log }=\frac{\ln \epsilon}{8^{4} \pi l^{3}} \int d^{6} x \sqrt{-\gamma^{0}}\left\{\frac{3}{50}\left(R^{0}\right)^{3}+R^{(0) i j} R^{(0) k l} R_{i j k l}^{0}-\frac{1}{2} R^{0} R^{(0) i j} R_{i j}^{0}\right. \\
\left.\quad+\frac{1}{5} R^{(0) i j} D_{i} D_{j} R^{0}-\frac{1}{2} R^{(0) i j} \square^{0} R_{i j}^{0}+\frac{1}{20} R^{0} \square^{0} R^{0}\right\} .
\end{aligned}
$$

In Eqs. (19) and (20) $R^{0}$ and $R^{(0) i j}$ are the Ricci scalar and Ricci tensor of the leading order metric $\gamma^{0}$ in the following expansion:

$$
\gamma_{i j}=\gamma_{i j}^{0}+x^{2} \gamma_{i j}^{2}+x^{4} \gamma_{i j}^{4}+\ldots
$$

and $D_{i}$ is the covariant derivative constructed by the leading order metric $\gamma^{0}$. Also, one should note that the inclusion of matter fields in the gravitational action produces an additional logarithmic divergence in the action for even $n$. This logarithmic divergence for $n=4$ and $n=6$ are [23]

$$
I_{\log }^{\mathrm{em}}=\frac{\ln \epsilon}{64 \pi l} \int d^{4} x \sqrt{\gamma^{0}} F^{(0) i j} F_{i j}^{0},
$$




$$
\begin{aligned}
I_{\log }^{\mathrm{em}}=\frac{\ln \epsilon}{8 \pi l^{3}} \int d^{6} x \sqrt{-\gamma^{0}}\{ & \frac{1}{16} R^{0} F^{(0) i j} F_{i j}^{0}-\frac{1}{8} R^{(0) i j} F_{i}^{(0) l} F_{j l}^{0} \\
& \left.+\frac{1}{64} F^{(0) i j}\left(D_{j} D^{k} F_{k i}^{0}-D_{i} D^{k} F_{k j}^{0}\right)\right\} .
\end{aligned}
$$

where $F_{i j}^{0}$ is the leading term of the electromagnetic field on the conformal boundary. All the contractions in Eqs. (22) and 23) should be done by the leading order metric $\gamma_{i j}^{0}$. In $4<n<7$ the matter field will cause a power law divergence in the action, which can be removed by a counterterm of the form [23]

$$
I_{c t}^{\mathrm{em}}=\frac{1}{256 \pi} \int d^{n} x \sqrt{-\gamma} \frac{(n-8)}{(n-4)} \Theta(n-5) F^{i j} F_{i j} .
$$

Thus, the total action can be written as a linear combination of the gravity term (11) and the counterterms (18) and (24). For the charged rotating and travelling magnetic branes investigated in this paper the counterterm $I_{\mathrm{ct}}^{\mathrm{em}}$ is zero and therefore the total action is

$$
I=I_{G}+I_{\mathrm{ct}}
$$

Having the total action, one can use the Brown and York definition [24] to construct a divergence-free stress-energy tensor as

$$
\begin{aligned}
T^{a b}= & \frac{1}{8 \pi}\left\{\left(K^{a b}-K \gamma^{a b}\right)-\frac{n-1}{l} \gamma^{a b}+\frac{l}{n-2}\left(R^{a b}-\frac{1}{2} R \gamma^{a b}\right)\right. \\
& +\frac{l^{3} \Theta(n-5)}{(n-4)(n-2)^{2}}\left[-\frac{1}{2} \gamma^{a b}\left(R^{c d} R_{c d}-\frac{n}{4(n-1)} R^{2}\right)-\frac{n}{(2 n-2)} R R^{a b}\right. \\
& \left.\left.+2 R_{c d} R^{a c b d}-\frac{n-2}{2(n-1)} \nabla^{a} \nabla^{b} R+\nabla^{2} R^{a b}-\frac{1}{2(n-1)} \gamma^{a b} \nabla^{2} R\right]+\ldots\right\} .
\end{aligned}
$$

The above stress tensor is divergence-free for $n \leq 6$, but we can always add more counterterms to have a finite action in higher dimensions (see, e.g., 25]).

The conserved charges associated to a Killing vector $\xi^{a}$ is

$$
\mathcal{Q}(\xi)=\int_{\mathcal{B}} d^{n} x \sqrt{\sigma} n^{a} T_{a b} \xi^{b},
$$

where $\sigma$ is the determinant of the metric $\sigma_{i j}$, appearing in the Arnowitt-Deser-Misner-like decomposition of the boundary metric,

$$
d s^{2}=-N^{2} d t^{2}+\sigma_{i j}\left(d x^{i}+N^{i} d t\right)\left(d x^{j}+N^{j} d t\right)
$$

In Eq. (28), $N$ and $N^{i}$ are the lapse and shift functions, respectively. For boundaries with timelike Killing vector $(\xi=\partial / \partial t)$, rotational Killing vector field $\left(\zeta_{i}=\partial / \partial \phi^{i}\right)$, and 
translational Killing vector $\left(\varsigma_{i}=\partial / \partial x^{i}\right)$ one obtains the conserved mass, angular momentum and linear momentum of the system enclosed by the boundary $\mathcal{B}$. In the context of AdS/CFT correspondence, the limit in which the boundary $\mathcal{B}$ becomes infinite $\left(\mathcal{B}_{\infty}\right)$ is taken, and the counterterm prescription ensures that the action and conserved charges are finite. No embedding of the surface $\mathcal{B}$ into a reference spacetime is required and the quantities which are computed are intrinsic to the spacetimes.

For our case, horizonless rotating spacetimes, the first Killing vector is $\xi=\partial / \partial t$ and therefore its associated conserved charge is the total mass of the system enclosed by the boundary given by

$$
M=\int_{\mathcal{B}} d^{n-1} x \sqrt{\sigma} T_{a b} n^{a} \xi^{b}=\frac{V_{n-1}}{8 \pi} m l^{p-1}\left[n\left(\Xi^{2}-1\right)+1\right],
$$

where $V_{n-1}$ denotes the volume of the hypersurface boundary $\mathcal{B}$ at constant $t$ and $r$, and $p$ is the number of angular coordinates $\phi^{i}$ of the spacetime. One may note that in the case of a spacetime with a longitudinal magnetic field, the numbers of angular coordinates and rotation parameters are the same, but for a spacetime with an angular magnetic field, they are different.

For the case of spacetimes with a longitudinal magnetic field, the charges associated with the rotational Killing symmetries generated by $\zeta_{i}=\partial / \partial \phi^{i}$ are the components of total angular momentum of the system enclosed by the boundary given as

$$
J_{i}=\int_{\mathcal{B}} d^{n-1} x \sqrt{\sigma} T_{a b} n^{a} \zeta_{i}^{b}=\frac{V_{n-1}}{8 \pi} n \Xi l^{p-1} m a_{i}
$$

In the case of the spacetimes with an angular magnetic field introduced in Sec. II E, we encounter conserved quantities associated with translational Killing symmetries generated by $\varsigma_{i}=\partial / \partial x^{i}$. These conserved quantities are the components of linear momentum calculated as

$$
P_{i}=\int_{\mathcal{B}} d^{n-1} x \sqrt{\sigma} T_{a b} n^{a} \varsigma_{i}^{b}=\frac{V_{n-1}}{8 \pi} n \Xi l^{p-2} m v_{i} .
$$

Now we show that the $r$ divergence of the total action can be removed by the counterterm method. We first calculate the logarithmic divergences due to the Weyl anomaly and matter field given in Eqs. (19), (201), (22), and (23). The leading metric $h_{i j}^{0}$ can be obtained as

$$
h_{i j}^{0} d x^{i} d x^{j}=-\frac{1}{l^{2}} d t^{2}+d \Omega^{2}+d X^{2} .
$$


Therefore the curvature scalar $R^{0}\left(h^{0}\right)$ and Ricci tensor $R_{i j}^{0}\left(h^{0}\right)$ are zero. Also, it is easy to show that $F_{i j}^{0}$ in Eqs. (22) and (23) vanishes. Thus, all the logarithmic divergences for the $(n+1)$-dimensional solutions given in Sec. II] are zero. It is also a matter of calculation to show that the counterterm action due to the electromagnetic field in Eq. (24) is zero. Using Eqs. (11), (18), and (25), one can show that the $r$ divergence of the action will be removed.

Next, we calculate the electric charge of the solutions. To determine the electric field we should consider the projections of the electromagnetic field tensor on special hypersurfaces. The normal to such hypersurfaces for the spacetimes with a longitudinal magnetic field is

$$
u^{0}=\frac{1}{N}, \quad u^{r}=0, \quad u^{i}=-\frac{N^{i}}{N}
$$

and the electric field is $E^{\mu}=g^{\mu \rho} F_{\rho \nu} u^{\nu}$. Then the electric charge $Q$ can be found by calculating the flux of the electromagnetic field at infinity, yielding

$$
Q=\frac{V_{n-1}}{4 \pi} \sqrt{\Xi^{2}-1} l^{p-2} q
$$

Note that the electric charge is proportional to the rotation parameter or boost parameter, and is zero for the case of a static solution.

\section{CLOSING REMARKS}

In this paper, we introduced two classes of solutions of Einstein-Maxwell gravity which are asymptotically anti-de Sitter. The first class of solutions yields a rotating spacetime with a longitudinal magnetic field. We found that these solutions have no curvature singularity and no horizons, but have conic singularity at $r=0$. In these spacetimes, when all the rotation parameters are zero (static case), the electric field vanishes, and therefore the brane has no net electric charge. For the spinning brane, when one or more rotation parameters are nonzero, the brane has a net electric charge which is proportional to the magnitude of rotation parameter given by $\sum_{i}^{k} a_{i}^{2}$. The second class of solutions yields a spacetime with angular magnetic field. These solutions have no curvature singularity, no horizon, and no conic singularity. Again we found that the branes in these spacetimes have no net electric charge when all the boost parameters are zero. We also showed that, for the case of traveling branes with one or more nonzero boost parameters, the net electric charge of the brane is proportional to the magnitude of the velocity of the brane $\left(\sum_{i}^{\kappa} v_{i}^{2}\right)$. 
We also used the counterterm method inspired by the AdS/CFT correspondence conjecture and calculated the conserved quantities of the two classes of solutions. We found that the logarithmic divergencies associated with the Weyl anomalies and matter field are zero, and showed that the $r$ divergence of the action is removed by use of the counterterm method.

\section{APPENDIX}

In this appendix, we want to show that the spacetime described by the metric (8) is geodesically complete for $r \geq 0$ 19, 26]. In fact, we want to show that every null or timelike geodesic starting from an arbitrary point can either extend to infinite values of the affine parameter along the geodesic or end on a singularity at $r=0$. To do this, we first perform the rotation boost $(\Xi t-a \phi) \mapsto t ;\left(a t-\Xi l^{2} d \phi\right) \mapsto l^{2} d \phi$ in the $t-\phi$ plane. Then the metric (8) becomes

$$
d s^{2}=-\frac{r^{2}+r_{+}^{2}}{l^{2}} d t^{2}+\frac{r^{2}}{\left(r^{2}+r_{+}^{2}\right) f(r)} d r^{2}+l^{2} f(r) d \phi^{2}+\frac{r^{2}+r_{+}^{2}}{l^{2}} d X^{2} .
$$

Using the geodesic equation, one obtains

$$
\begin{aligned}
& \dot{t}=\frac{l^{2}}{r^{2}+r_{+}^{2}} E, \quad \dot{x^{i}}=\frac{l^{2}}{r^{2}+r_{+}^{2}} P^{i}, \quad \dot{\phi}=\frac{1}{l^{2} f(r)} L \\
& r^{2} \dot{r}^{2}=\left(r^{2}+r_{+}^{2}\right) f(r)\left[\frac{l^{2}\left(E^{2}-\mathbf{P}^{2}\right)}{r^{2}+r_{+}^{2}}-\alpha\right]-\frac{r^{2}+r_{+}^{2}}{l^{2}} L^{2},
\end{aligned}
$$

where the overdot denotes the derivative with respect to an affine parameter and $\alpha$ is zero for null geodesics and +1 for timelike geodesics. $E, L$, and $P^{i}$ are the conserved quantities associated with the coordinates $t, \phi$, and $x^{i}$ respectively, and $\mathbf{P}^{2}=\sum_{i=1}^{n-2}\left(P^{i}\right)^{2}$. Notice that $f(r)$ is always positive for $r>0$ and zero for $r=0$.

First we consider the null geodesics $(\alpha=0)$. (i) If $E^{2}>\mathbf{P}^{2}$ the spiraling particles $(L>0)$ coming from infinity have a turning point at $r_{t p}>0$, while the nonspiraling particles $(L=0)$ have a turning point at $r_{t p}=0$. (ii) If $E=\mathbf{P}$ and $L=0$, whatever is the value of $r, \dot{r}$ and $\dot{\phi}$, vanish and therefore the null particles move in a straight line in the $(n-2)$-dimensional submanifold spanned by $x^{1}$ to $x^{n-2}$. (iii) For $E=\mathbf{P}$ and $L \neq 0$, and also for $E^{2}<\mathbf{P}^{2}$ and any value of $L$, there is no possible null geodesic.

Now, we analyze the timelike geodesics $(\alpha=+1)$. A timelike geodesic is possible only if $l^{2}\left(E^{2}-\mathbf{P}^{2}\right)>r_{+}^{2}$. In this case spiraling $(L \neq 0)$ timelike particles are bound between $r_{t p}^{a}$ 
and $r_{t p}^{b}$ given by

$$
0<r_{t p}^{a} \leq r_{t p}^{b}<\sqrt{l^{2}\left(E^{2}-\mathbf{P}^{2}\right)-r_{+}^{2}}
$$

while the turning points for the nonspiraling particles $(L=0)$ are $r_{t p}^{1}=0$ and $r_{t p}^{2}=$ $\sqrt{l^{2}\left(E^{2}-\mathbf{P}^{2}\right)-r_{+}^{2}}$. Thus, we have confirmed that the spacetime described by Eq. (8) is both null and timelike geodesically complete.

[1] J. Maldacena, Adv. Theor. Math. Phys. 2, 231 (1988); E. Witten, ibid. 2, 253 (1998); O. Aharony, S. S. Gubser, J. Maldacena, H. Ooguri, and Y. Oz, Phys. Rep. 323, 183 (2000).

[2] E. Witten, Adv. Theor. Math. Phys. 2, 505 (1998).

[3] M. Hennigson and K. Skenderis, J. High Energy Phys. 7, 023 (1998).

[4] V. Balasubramanian and P. Kraus, Commun. Math. Phys. 208, 413 (1999).

[5] S. Nojiri and S. D. Odintsov, Phys. Lett. B 444, 92 (1998); S. Nojiri, S. D. Odintsov, and S. Ogushi, Phys. Rev. D 62, 124002 (2000).

[6] M. H. Dehghani, Phys. Rev. D 66, 044006 (2002).

[7] M. H. Dehghani, Phys. Rev. D 65, 124002 (2002); R. G. Cai, ibid. 63, 124018 (2001); A. M. Ghezelbash D. Ida, R. B. Mann, and T. Shiromizu, Phys. Lett. B 535, 315 (2002).

[8] M. H. Dehghani and R. B. Mann, Phys. Rev. D 64, 044003 (2001); M. H. Dehghani, ibid. 65, 104030 (2002); M. H. Dehghani and H. KhajehAzad, Can. J. Phys. 81, 1363 (2003).

[9] V. Balasubramanian, P. Horova, and D. Minic, J. High Energy Phys. 05, 043 (2001); M. H. Dehghani, Phys. Rev. D 65, 104003 (2001).

[10] R. B. Mann, Class. Quantum Grav. 14, L109 (1997); W. L. Smith and R. B. Mann, Phys. Rev. D 56, 4942 (1997); C. G. Huang, C-B Liang, Phys. Lett. A 201, 27 (1995); L. Vanzo, Phys. Rev. D 56, 6475 (1997); S. Aminneborg I. Bengtsson, S. Holst, and P. Peldan, Class. Quantum Grav. 13, 2707 (1996); R. G. Cai and Y. Z. Zhang, ibid. 54, 4891 (1996); R. G. Cai, J. Y. Ji, and K. S. Soh, ibid. 57, 6547 (1998); D. Klemm, J. High Energy Phys. 11, 019 (1998).

[11] D. Klemm, V. Moretti, and L. Vanzo, Phys. Rev. D 57, 6127 (1998); D. Klemm, J. High Energy Phys. 11, 019 (1998).

[12] J. P. S. Lemos, Class. Quantum Grav. 12, 1081 (1995); Phys. Lett. B 353, 46 (1995); J. P. S. Lemos and V. T. Zanchin, Phys. Rev. D 54, 3840 (1996). 
[13] A. M. Awad, Clas. Quantum Grav. 20, 2827 (2003); M. H. Dehghani and A. KhoddamMohammadi, Phys. Rev. D 67, 084006 (2003).

[14] M. H. Dehghani, Phys. Rev. D 67, 064017, (2003).

[15] M. Cvetic and D. Youm, Nucl. Phys. B499, 253 (1997); K. Behrndt, M. Cvetic, and W. A. Sabra, ibid. B553, 317 (1999); M. Cvetic and S. S. Gubser, J. High Energy Phys. 04, 024 (1999).

[16] T. Levi-Civita, Atti Accad. Naz. Lincei, Cl. Sci. Fis., Mat. Nat., Rend. 283 (1919); L. Marder, Proc. R. Soc. London A244, 524 (1958).

[17] A. Vilenkin, Phys. Rev. D 23, 852 (1981); W. A. Hiscock, ibid. 31, 3288 (1985); D. Harari and P. Sikivie, ibid. 37, 3438 (1988); J. R. Gott, Astrophys. J. 288, 422 (1985); A. D. Cohen and D. B. Kaplan, Phys. Lett. B215, 65 (1988); R. Gregory, ibid. 215, 663 (1988); A. Banerjee, N. Banerjee, and A. A. Sen, Phys. Rev. D 53, 5508 (1996).

[18] B. C. Mukherji, Bull. Calcutta. Math. Soc. 30, 95 (1938); W. B. Bonnor, Proc. Roy. S. London A67, 225 (1954); J. L. Safko, Phys. Rev. D 16, 1678, (1977); E. Witten, Nucl. Phys. B249, $557(1985)$.

[19] O. J. C. Dias and J. P. S. Lemos, Class. Quantum Grav. 19, 2265 (2002).

[20] O. Lunin and S. D. Mathur, Nucl. Phys. B623, 342 (2002); O. Lunin, J. Maldacena, and L. Maoz, hep-th/0212210.

[21] W. A. Sabra, Phys. Lett. B 545, 175 (2002); J. T. Liu and W. A. Sabra, hep-th/0307300.

[22] R. Emparan, C. V. Johnson, and R. C. Myers, Phys. Rev. D 60, 104001 (1999); R. B. Mann, ibid. 60, 104047 (1999); 61, 084013 (2000); S. Das and R. B. Mann, J. High Energy Phys. 08, 033 (2000); A. M. Awad and C. V. Johnson, Phys. Rev. D 61, 084025 (2000).

[23] S. de Haro, K. Skenderis, and S. N. Solodukhin, Commun. Math. Phys. 217, 595 (2001); M. Bianchi, D. Z. Freedman, and K. Skenderis, Nucl. Phys. B631, 159 (2002); M. TaylorRobinson, hep-th/0002125.

[24] J. D. Brown and J. W. York, Phys. Rev. D 47, 1407 (1993).

[25] P. Kraus, F. Larsen, and R. Siebelink, Nucl. Phys. B563, 259 (1999).

[26] J. H. Horne and G. T. Horowitz, Nucl. Phys. B368, 444 (1992). 